\title{
QUALIDADE DE VIDA DE IDOSOS PRATICANTES DE EXERCÍCIO FÍSICO EM ACADEMIAS AO AR LIVRE EM PARQUE A CÉU ABERTO
}

Natalia Cristina Andrade ${ }^{1}$, Carlos Roberto Moreira Junior ${ }^{2}$, William Frederico Almeida Klebis ${ }^{2}$, Isabela Cristina Duarte Araújo ${ }^{1}$, Ana Clara Campagnolo Gonçalves Toledo ${ }^{1,2}$, Weber Gutemberg Alves de Oliveira ${ }^{1}$, Aline Duarte Ferreira $^{1,2}$

Universidade do Oeste Paulista - UNOESTE, ${ }^{1}$ Curso de Fisioterapia e ${ }^{2}$ curso de Educação Física, Presidente Prudente/SP. E-mail: nathandraade@outlook.com

\section{RESUMO}

O objetivo do estudo foi avaliar a percepção da qualidade de vida dos idosos praticantes de exercícios físicos em academias ao ar livre em um parque. A amostra foi composta por 39 idosos, divididos em dois grupos: $\mathrm{G} 1$ $(n=19)$ que realizavam caminhada e $G 2(n=20)$ que realizavam exercícios nas academias ao ar livre, ambos não supervisionados. $O$ nível de atividade física foi avaliado pelo questionário internacional de atividade física IPAQ e a percepção da qualidade de vida pelo questionário SF-36. A análise dos dados foi realizada com o programa SPSS $22.0(p<0,05)$. Não houve diferença significativa entre os grupos na comparação da percepção do estado geral de saúde, pois apresentaram um estado de saúde de excelente para boa e na comparação da qualidade de vida, alta pontuação. Concluiu-se que a prática de exercício físico, seja em academias ou caminhada, gera manutenção da saúde e qualidade de vida para idosos.

Palavras-chave: áreas verdes, exercício físico, idoso, qualidade de vida, academias de ginástica.

\section{LIFE QUALITY OF ELDERLY PEOPLE PRACTICING PHYSICAL ACTIVITY IN OPEN-AIR GYMS IN AN OPEN-AIR PARK}

\section{ABSTRACT}

The objective of this study was to assess the perception of the quality of life of elderly practitioners of physical activity in outdoors academies in a park. The sample was composted by 39 elderly, divided in two groups: $G 1(n=19)$ who was walking and $G 2(n=20)$ that realized exercises in outdoors academies, both without supervision. The activity level was evaluated by international physical activities questionnaire IPAQ and the perception of quality of life by SF-36 questionnaire. The data analysis was executed with SPSS 22.0 program $(p<0.05)$. There was no significant difference between the groups at comparison of general health status perception, because were presented an excellent to good health status and high score in quality life comparison. It was concluded that practice of physical exercises, both in walks or academies, result in health maintaining and quality of life for elderly people.

Keywords: green areas, physical exercise, elderly people, life quality, gymnastics academies.

\section{INTRODUÇÃO}

Academias ao ar livre são espaços que possibilitam a prática de exercício físico pela comunidade e com isso possui uma grande importância na relação com a saúde dos praticantes e da comunidade próxima a esse espaço ${ }^{1}$. A prática de exercício físico é considerada uma estratégia de prevenção de doenças, sendo capaz de retardar incapacidades funcionais e melhorar a condição neuromotora, musculoesquelética e cardiorrespiratória ${ }^{2}$.

O envelhecimento é um processo congênito e ininterrupto da vida de todo ser vivo, ou seja, é um processo universal ${ }^{3}$. Caracterizado por ser um fenômeno complexo e variável, marcado pela gradativa redução das funções orgânicas, associado com um declínio da capacidade funcional do sistema neuromuscular, 
endócrino, cardiovascular, ósseo e respiratório, o que favorece a redução funcional da força física ${ }^{4,5}$.

A prática de exercícios físicos regulares de idosos e não qualquer atividade física é a responsável pela melhora no estado de saúde e na proteção do organismo contra as doenças crônicas degenerativas ${ }^{6}$. 0 exercício físico estimula as estruturas do corpo, sendo imposta uma sobrecarga no organismo, que assim se adapta em forma e função como resposta às sobrecargas que lhes são impostas, ou seja, adaptações orgânicas e fisiológicas que tornam o organismo mais apto para a realização do exercício, sendo necessários sempre novos estímulos de formas variadas para obter novas adaptações ${ }^{7}$.

A prática de exercícios físicos regulares em parques e academias a céu aberto tem como fatores positivos associados à realização ao ar livre incluindo: estar em um cenário agradável; o incentivo por estar praticando exercício físico ao lado de outras pessoas; a facilidade de acesso a esses locais e a satisfação pessoal. Além de proporcionar benefícios físicos para uma diminuição dos riscos de doenças metabólicas, as academias ao ar livre promovem, ainda, benefícios psicológicos, tais como a redução do estresse, a sensação de bem-estar e liberdade, pelo fato de ser um ambiente natural ${ }^{8}$.

Entende-se por qualidade de vida a percepção do indivíduo da sua posição na vida, no contexto da cultura e sistemas de valores nos quais se insere e em relação aos seus objetivos, expectativas, padrões e preocupações. Trata-se de um amplo conceito de classificação, afetado, de modo complexo, pela saúde física do indivíduo, estado psicológico, relações sociais, nível de independência e pelas suas relações com as características mais relevantes do seu meio ambiente ${ }^{9}$.

O exercício físico regular melhora a qualidade e expectativa de vida do idoso, devendo ser estimulada ao longo de sua vida ${ }^{10,11}$. Programas e ações públicas de exercícios físicos específicos, que são voltadas para a população idosa, representam também um desafio, pois deve ser levada em conta uma preparação dos profissionais, a sensibilização da população, a implementação das atividades e também a existência de políticas públicas juntamente com o apoio dos gestores ${ }^{12,13}$.

Justifica-se esta pesquisa pela necessidade de compreender os possíveis benefícios dos exercícios físicos sobre a percepção da qualidade de vida em idosos, tanto por meio da caminhada quanto nas academias em parques a céu aberto. Assim, o objetivo deste estudo foi avaliar a percepção da qualidade de vida de idosos que praticam exercícios físicos nas academias ao ar livre e comparar com idosos que realizam exercícios físicos em parques a céu aberto.

\section{METODOLOGIA}

A pesquisa foi aprovada pelo Comitê de Ética e Pesquisa da Universidade do Oeste Paulista (CAAE 69498717.6.0000.5515/3931). Todos os idosos foram informados sobre os procedimentos da pesquisa, e a mesma só foi realizada, após assinatura do Termo de Consentimento Livre e Esclarecido, conforme Resolução CONEP 466/2012.

Participaram do estudo, 39 idosos, frequentadores de um parque a céu aberto da cidade de Presidente Prudente/SP. Destes, 19 idosos eram praticantes da caminhada como exercício físico, enquanto 20 eram praticantes dos exercícios físicos em academias ao ar livre no mesmo parque.

Como critérios de inclusão, apenas participaram do estudo, idosos com idade igual ou superior a 60 anos, ambos os gêneros e com no mínimo seis meses de exercício físico regular. A coleta de dados foi realizada em dois períodos, manhã e tarde no próprio local de exercícios de ambos os grupos.

Para a avaliação da percepção da qualidade, foi aplicado o questionário genérico SF36 , que contém 36 itens que abrangem oito domínios: capacidade funcional (CF), aspectos físicos (AF), dor (DOR), estado geral de saúde (EGS), vitalidade (VIT), aspecto social (AS), aspecto emocional (AE) e saúde mental (SM). O escore varia de $0-100(0=$ pior $Q V R S ; 100=$ melhor QVRS) ${ }^{14}$.

Para avaliar o nível de atividade física foi utilizado o questionário internacional de atividade física, o IPAQ, que visa saber que tipos de atividade física as pessoas fazem como parte do seu dia a dia. As perguntas são relacionadas ao tempo gasto fazendo atividade física na última semana, incluem as atividades corriqueiras como as que você faz no trabalho, para ir de um lugar a outro, por lazer, por esporte, por exercício ou como serviços de casa ${ }^{15}$.

A descrição dos dados quantitativos foi apresentada em forma de mediana e intervalo 
interquartílico (25-75\%), uma vez que não passaram pelo teste de normalidade de ShapiroWilk. Os dados qualitativos foram apresentados em frequência e percentual. A comparação entre os grupos foi realizada por meio da análise de covariância (ANCOVA) ajustado para idade, sexo, escolaridade, histórico ocupacional e nível de atividade física para variáveis quantitativas, exceto para a comparação da idade que foi realizado por meio do teste de Mann-Whitney. Na comparação entre os grupos para dados qualitativos foi utilizado teste qui-quadrado com correção de Fisher para tabelas cruzadas $2 \times 2$ ou teste de associação linear por linear para tabelas cruzadas acima de $2 \times 2$. Para todos os teste foi adotado nível de significância de $5 \%(p<0,05)$. A análise dos dados foi realizada com o programa SPSS 22.0.

\section{RESULTADOS}

As características gerais da população do estudo são apresentadas na Tabela 1. Nota-se que não houve diferença significativa entre os grupos para as variáveis sexo, idade, escolaridade e histórico ocupacional.

Tabela 1. Análise descritiva da caracterização da amostra em estudo ( $n=39)$.

\begin{tabular}{l|c|c|c}
\hline & $\begin{array}{c}\text { Caminhada } \\
(\mathrm{n}=19)\end{array}$ & $\begin{array}{c}\text { Academia ar livre } \\
(\mathrm{n}=20)\end{array}$ & $\boldsymbol{p}$ \\
\hline Sexo (F/M), $\mathrm{n}$ & $\begin{array}{c}12 / 7 \\
13 / 7\end{array}$ & $0,905^{\mathrm{b}}$ \\
Idade (anos), mediana (IQR) & $66,0(61,0-72,0)$ & $65,5(61,0-67,0)$ & $0,411^{\mathrm{a}}$ \\
\hline Escolaridade, $n(\%)$ & $9(47)$ & $7(35)$ & \\
Ensino fundamental & $6(32)$ & $10(50)$ & $0,790^{\mathrm{c}}$ \\
Ensino médio & $4(21)$ & $3(15)$ & \\
Ensino superior & $2(11)$ & $2(10)$ & $1^{\mathrm{b}}$ \\
\hline Histórico ocupacional, $\boldsymbol{n ( \% )}$ & $17(89)$ & $18(90)$ & \\
Sim & & & \\
Não &
\end{tabular}

Legenda: F/M: feminino/masculino. Dados quantitativos expressos em mediana e intervalo interquartílico $25-75 \%$ (IQR). Dados qualitativos expressos em frequência (n) e percentual (\%). ${ }^{\mathrm{a}}$ Teste de Mann-Whitney; ${ }^{\mathrm{b}}$ Qui-quadrado com correção de Fisher; 'Associação linear por linear; ${ }^{*} p<0.05$

A Figura 1 apresenta o nível de atividade física, avaliado por meio do IPAQ entre os grupos, onde a maioria dos idosos foram classificados como ativos (68\% do grupo caminhada e $80 \%$ do grupo academia ao ar livre; $p=0,480$ ).

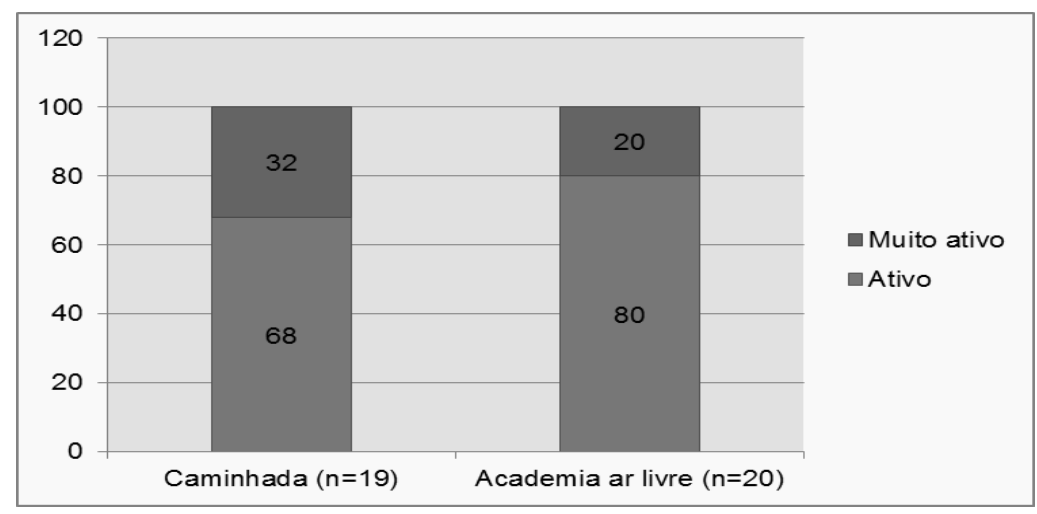

Figura 1. Nível de atividade física (IPAQ) entre os grupos de idosos. Qui-quadrado com correção de Fisher $(p=0,480)$. Dados expressos em porcentagem.

Não houve diferença significativa entre os grupos na comparação da percepção do estado geral de saúde, pois todos os idosos avaliados reportaram apresentar um estado de saúde de excelente para boa, sendo que nenhum deles disse apresentar estado de saúde ruim (Tabela 2). 
Tabela 2. Comparação da percepção do estado geral de saúde entre os grupos de idosos de acordo com o ambiente que praticam exercícios físicos.

\begin{tabular}{lccc}
\hline Percepção do EGS, $\boldsymbol{n}(\%)$ & $\begin{array}{c}\text { Caminhada } \\
(\mathrm{n}=19)\end{array}$ & $\begin{array}{c}\text { Academia ar livre } \\
(\mathrm{n}=20)\end{array}$ & $\boldsymbol{p}$ \\
\hline Excelente & $6(31)$ & $4(20)$ & \\
Muito Boa & $6(31)$ & $3(15)$ & \\
Boa & $5(26)$ & $11(55)$ & $0,215^{\mathrm{a}}$ \\
Regular & $2(11)$ & $2(10)$ & \\
Ruim & $0(0)$ & $0(0)$ & \\
\hline
\end{tabular}

Legenda: EGS: estado geral de saúde. Dados qualitativos expressos em frequência (n) e percentual (\%). ${ }^{2}$ Associação linear por linear.

Na comparação da qualidade de vida, todos os idosos avaliados apresentaram alta pontuação no questionário SF-36, não observando diferença significativa entre os grupos avaliados mesmo quando ajustados para fatores de confusão como idade, sexo, escolaridade, histórico ocupacional e nível de atividade física.

Tabela 3. Comparação da qualidade de vida entre os grupos de idosos de acordo com o ambiente que praticam exercícios físicos.

\begin{tabular}{l|c|c|c}
\hline & Caminhada $(\mathrm{n}=19)$ & $\begin{array}{c}\text { Academia ar livre } \\
(\mathrm{n}=20)\end{array}$ & $\boldsymbol{p}$ \\
\hline CF & $85,0(80,0-90,0)$ & $90,0(71,3-93,8)$ & 0,821 \\
AF & $100,0(100,0-100,0)$ & $100,0(56,3-100,0)$ & 0,088 \\
DOR & $72,0(51,0-100,0)$ & $79,0(61,3-100,0)$ & 0,368 \\
EGS & $72,0(67,0-82,0)$ & $72,0(62,0-80,0)$ & 0,267 \\
VIT & $80,0(65,0-100,0)$ & $80,0(65,0-85,0)$ & 0,323 \\
AS & $100,0(100,0-100,0)$ & $100,0(90,7-100,0)$ & 0,836 \\
AE & $100,0(100,0-100,0)$ & $100,0(100,0-100,0)$ & 0,171 \\
SM & $88,0(80,0-96,0)$ & $84,0(73,0-92,0)$ & 0,885 \\
\hline
\end{tabular}

Legenda: Dados quantitativos expressos em mediana e intervalo interquartílico 25-75\% (IQR). CF: capacidade funciona; AF: aspecto físico; EGS: estado geral de saúde; VIT: vitalidade; AS: aspecto social; AE: aspecto emocional; SM: saúde mental. Teste de ANCOVA ajustado para idade, sexo, escolaridade, histórico ocupacional e nível de atividade física.

Na comparação dos domínios da qualidade de vida entre os grupos, a capacidade funcional e a dor, obteve um melhor resultado no grupo de idosos que praticavam academia ao ar livre, não havendo diferença significativa entre os grupos avaliados.

\section{DISCUSSÃO}

O presente estudo teve por finalidade avaliar a percepção da qualidade de vida de idosos que praticam exercício físico regular em academias ao ar livre e compará-los com idosos que fazem caminhada em parques a céu aberto como exercício físico. A percepção da qualidade de vida apresentou alta pontuação em ambos os grupos avaliados, evidenciando que a prática de exercício físico influencia a qualidade de vida da população idosa ${ }^{16}$. Em estudo europeu com população idosa representativa da Espanha, observou-se que idosos envolvidos em exercícios físicos apresentaram escores mais altos em praticamente todos os domínios do SF-36 ${ }^{17}$.

Entretanto, quando comparado os aspectos de capacidade funcional e dor, o grupo da academia ao ar livre obteve melhores resultados, pelo fato de que a academia ao ar livre possibilita que o usuário exercite tantos os grupos musculares superiores quanto os inferiores. Em estudo de caso realizado por Lima et al. ${ }^{18}$ evidenciaram que os exercícios físicos realizados em academias ao ar livre auto selecionados são suficientes para o estímulo a um meio de vida mais ativo, como também para gerar condicionamento ao idoso, pois assemelham-se a percepções de esforço próximo aos limites recomendados de um exercício regular.

0 estudo de Marcelino et al. $^{19}$ observaram que no decorrer da aplicação de 
exercícios resistidos com o uso de aparelhos musculares, houve uma melhora em comportamentos funcionais como ainda nos aspectos da análise das percepções físicas.

No estudo de Silva et al. ${ }^{20}$ que compararam os níveis de atividade física e qualidade de vida entre idosos que praticavam exercícios físicos regulares e idosos sedentários, concluíram que o nível de atividade física nem sempre está diretamente relacionado a prática de exercício físico propriamente dito, pois outros fatores podem interferir na classificação final, como as atividades diárias realizadas em casa no seu cotidiano. Constataram ainda, que os idosos que praticavam exercícios físicos obtiveram melhor índice de qualidade de vida, indicando que a prática de exercício físico pode vir a ser determinante para a melhoria da qualidade de vida.

Sendo um potencial fator de risco, a ausência da prática de exercício físico regularmente pode levar a um aumento no declínio funcional e os custos com tratamentos. Pensando nisso, a implantação de programas de exercícios físicos pode vir a ser uma estratégia eficaz nos efeitos da dor, no aumento da capacidade funcional, energia, vitalidade, dentre outros aspectos físicos ${ }^{21,22}$.

Portanto, é importante que ações voltadas à prática de exercícios físicos não sejam apenas para a ocupação de tempo livre dos idosos. É preciso que novas estratégias sejam criadas para estimular a prática de exercícios físicos tanto em academias ao ar livre quanto realização da caminhada de forma regular.

Dessa forma, concluímos que a prática de exercício físico regular na terceira idade, seja em academias ao ar livre como na caminhada, é capaz de promover uma manutenção da saúde e qualidade de vida de idosos.

\section{CONFLITO DE INTERESSE}

Os autores declaram não haver qualquer potencial conflito de interesse que possa interferir na imparcialidade deste trabalho científico.

\section{REFERÊNCIAS}

1. Souza CA, Fermino RC, Añez CRR, Reis RS. Perfil dos frequentadores e padrão de uso das academias ao ar livre em bairros de baixa e alta renda de Curitiba-PR. Rev Bras Ativ Fis Saúde. 2014;19(1):86-97.

DOI:
2. Coelho CF, Burini RC. Atividade física para prevenção e tratamento das doenças crônicas não transmissíveis e da incapacidade funcional. Rev Nutr. 2009;22(6):937-946. DOI:

https://doi.org/10.1590/S1415$\underline{52732009000600015}$

3. Pelegrino, Paulo Sergio. Perspectiva biopsicológica do envelhecimento [coordenação geral Áurea Eleotério Soares Barroso]. - São Paulo: Secretaria Estadual de Assistência e Desenvolvimento Social: Fundação Padre Anchieta, 2009.

4. Torres AC, Brito F, Castro MAS, Filho PJ, Borragine SOF. Exercícios resistidos: benefícios da prática sistemática em 19 idosos. Rev Digital, Buenos Aires. 2010; 15(148).

5. Vieira AGS, Schettino L, Machado M, Pereira R. Análise da força e autonomia de idosas: relação ente idade e performance musculoesquelética. RBCEH, Passo Fundo. 2009;5(6):225-232. DOI: http://dx.doi.org/10.5335/rbceh.2009.02

6. Sabá F. Mexa-se - Atividade física: saúde e bemestar. 3.ed. São Paulo: Phorte; 2011.

7. Barbosa AR. Treinamento contra resistência e função muscular em idoso. Rev Digital, Buenos Aires. 2007;12(108).

8. Sawada FT, Trajano GS, Nunes LFA, Malavazzi MA, Eger RF, Silva WA, Galo M. Análise espaço temporal do Parque do Povo de Presidente Prudente a partir de fotos aéreas e imagem orbital. [Trabalho de graduação do curso de engenharia cartográfica]. Presidente Prudente: Faculdade de Ciências e Tecnologia da Universidade Estadual Paulista; 2007.

9. The WHOQOL Group. The World Health Organization quality of life assessment WHOQOL: position paper from the World Health Organization. Soc Sci Med. 1995;41(10):1403-10. DOI: https://doi.org/10.1016/0277$\underline{9536(95) 00112-K}$

10. Vagetti GC, Filho VCB, Moreira NB, Oliveira V, Mazzardo O, Campos W. Condições de saúde e variáveis sociodemográficas associadas à qualidade de vida em idosas de um programa de atividade física de Curitiba, Paraná, Sul do Brasil. 
Cad Saúde Púb. 2013;29(5):955-69. DOI: https://doi.org/10.1590/S0102-

311X2013000500013

11. Maciel MG. Atividade física e funcionalidade do idoso. Motriz. 2010;16(4):1024-32. DOI: https://doi.org/10.5016/1980-

\subsection{0v16n4p1024}

12. Benedetti TRB, Gonçalves LHT, Mota JAPS. Uma proposta de política pública de atividade física para idosos. Texto Contexto Enferm. 2007;16(3):387-98.

DOI:

https://doi.org/10.1590/S0104-

\section{3}

13. Moretti AC, Almeida V, Westphal MF, Bógus CM. Práticas corporais/atividade física e políticas públicas de promoção da saúde. Saúde Soc. 2009;18(2):346-54. DOI:

https://doi.org/10.1590/S0104-

\section{$\underline{12902009000200017}$}

14. Ciconelli RM, Ferraz MB, Santos W, Meinão I, Quaresma MR. Tradução para a língua portuguesa e validação do questionário genérico de avaliação de qualidade de vida SF-36 (Brasil SF-36). Rev Bras Reumatol. 1999;39(3):143-50.

15. Benedetti TRB, Antunes PC, Añez CRR, Mazo GZ, Petroski EL. Reprodutibilidade e validade do Questionário Internacional de Atividade Física (IPAQ) em homens idosos. Rev Bras Med Esporte. 2007;13(1):11-16.

DOI:

https://doi.org/10.1590/S1517-

$\underline{86922007000100004}$

16. Silveira MM, Portuguez MW. A influência da atividade física na qualidade de vida de idosas. Ciên Saúde. 2017;10(4):220-25. DOI: https://doi.org/10.15448/1983-

652X.2017.4.26390

17. Castillon PG, Peralta PSO, Banegas JR, Lopez E, Artalejo FR. Physical activity and quality of life in older adults in Spain. Med Clin 2004;123(16):60610.

18. Lima FLR. Percepção do esforço em idosos nas academias ao ar livre. Rev Bras Prescr Fisiol Exerc. 2013;7(37):55-64.

19. Marcelino VR. A estruturação de um programa de trabalho resistido para o idoso: uma proposta de intervenção [dissertação]. Campinas: Universidade Estadual de Campinas; 2003.

20. Silva MF, Goulart NBA, Lanferdini FJ, Marcon $M$, Dias CP. Relação entre os níveis de atividade física e qualidade de vida de idosos sedentários e fisicamente ativos. Rev Bras Geriatr Gerontol. 2012;15(4):634-642.

DOI:

https://doi.org/10.1590/S1809-

$\underline{98232012000400004}$

21. Nelson ME, Rejeski WJ, Blair SN, Duncan PW, Judge JO, King AC, Macera CA, Sceppa CC. Physical activity and public health in older adults: recommendation from the American College of Sports Medicine and the American Heart Association. Med Sci Sports Exerc. 2007;39(8):1435-45. DOI: https://doi.org/10.1249/mss.0b013e3180616aa2

22. Stiggelbout M, Popkema D Y, Rock MH, Greef $M$, Mechelen WV. Once a week is not enough: effects of a widely implemented group based exercise programme for older adults. A randomized controlled trial. J Epidemiol Comm Health. 2004;58(2):83-8. DOI: https://doi.org/10.1136/jech.58.2.83

Recebido para publicação em 17/08/2018

Revisado em 27/08/2018

Aceito em 03/09/2018 